\title{
XXIII.
}

Aus dem pharmakologischen Institut der Universität zu Tokio.

\section{Ueber den wirksamen Bestandtheil der Adonis amurensis Reg. et Radd.}

\author{
Yon
}

Dr. med. Y. Inoko.

Bekanntlich hat Cervello $\left.{ }^{1}\right) 1882$ in der Adonis vernalis L. ein Glykosid entdeckt, welches von ihm Adonidin genannt wurde. Dieser Körper gehört pharmakologiseh zur Digitalingruppe und wird als Ersatzmittel für Digitalin, resp. Digitalis vielfach empfohlen. Später gelang es demselben Forseher, auch in der Adonis cupiana Guss. das Adonidin nachzuweisen. Es giebt in Japan eine einheimische Adonisart, Adonis amurensis Reg. et Radd. (japaniseh: Fukudjusō). Ueber diese Pflanze haben jüngst Dr. Y. Tawara und M. Yamamoto eingehende chemische Untersuchungen angestellt und Beide entdeckten ein Glykosid, welches zwar dem Adonidin ähnlich, aber von ihm deutlich verschieden ist. Da sie ihre Ergebnisse an anderem Orte ausfuhrlich berichten werden, so beschränke ich mich hier auf eine kurze Mittheilung von einigen chemisehen Verbältnissen. ${ }^{2}$ )

Das Glykosid ist amorph, farblos, bitter, löslich in Wasser, Alkohol und Chloroform, fast unlöslich in Aether. Es ist stickstofffrei und hat folgende procentische Zusammensetzung:

$$
\begin{aligned}
& \text { C } 60,81 \text { Proc. } \\
& \text { H } 8,60= \\
& \text { O } 30,59=
\end{aligned}
$$

Dureh Kochen mit verdünnten Säuren zerfällt es in Zucker und ejne harzartige, in Aether lösliche Substanz. Mit concentrirter Schwefelsäure und Brom entsteht keine bemerkenswerthe Reaction. ${ }^{3}$ )

1) Ueber den wirksamen Bestandtheil der Adonis vernalis L. Archiv f.exp. Patb. u. Pharm. XV. Bd. S. 235.

2) Nach mündlichem Bericht des Herrn Yamamoto.

3) Nach meiner eigenen Untersuchung. 
Ueber den wirksamen Bestandtheil der Adonis amurensis Reg. et Radd. 303

Durch die Güte der Herren Tawara und Yamamoto erhielt ich ein Quantum dieses Körpers in chemisch vollkommen reinem Zustand und unterzog denselben einer pharmakologischen Prüfung. Dieselbe ergab, dass das Glykosid zwar qualitativ dem Adonidin völlig analog wirkt, aber quantitativ davon erheblich abweicht. Infolge dessen schlagen wir für diese Substanz den Namen "Adonin" vor. Das harzartige Zersetzungsproduct erwies sich pharmakologiseh als unwirksam. ${ }^{1}$ )

Was nun die Wirkung des Adonin auf das Froschberz anbetrifft, so sind die einzelnen Symptome, wie Verstärkung der systolischen Contraction, Verlangsamung der Frequenz, Peristaltik und systoliseher Stillstand, genan wie beim Digitalin, resp. Adonidin und bedürfen keiner näheren Auseinandersetzung. Nur die Dosen, welche zum systolischen Stillstand führen, sind bemerkenswerth. Im Folgenden gebe ich eine tabellarische Zusammenstellung der einzelnen Versuche, welche ich im Juni 1890 angestellt habe.

\begin{tabular}{|c|c|c|c|c|c|c|c|c|}
\hline 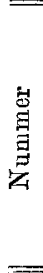 & 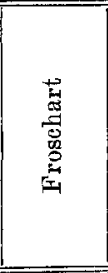 & 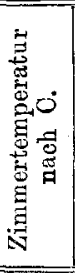 & 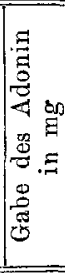 & 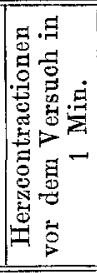 & 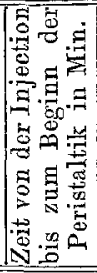 & 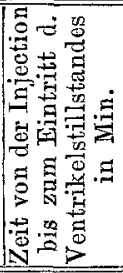 & 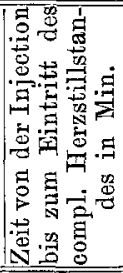 & Bemerkungen \\
\hline 1 & R. escul. & $21^{\circ}$ & 1,4 & 73 & 53 & - & - & $\begin{array}{l}\text { Nach } 1 \text { Stunde kein Still- } \\
\text { stand. }\end{array}$ \\
\hline 2 & $=$ & $22^{0}$ & 1,4 & 75 & - & - & - & $\begin{array}{l}\text { Nach } 1 \text { Stunde weder Peri- } \\
\text { staltik noch Stillstand. }\end{array}$ \\
\hline 3 & $=$ & $22^{0}$ & 2,1 & 72 & 33 & - & - & $\begin{array}{l}\text { Nach } 1 \text { Stunde kein Still- } \\
\text { stand. }\end{array}$ \\
\hline 4 & $=$ & $20,5^{0}$ & 2,1 & 56 & - & - & - & $\begin{array}{l}\text { Nach } 1 \text { Stunde weder Peri- } \\
\text { staltik noch Stillstand. }\end{array}$ \\
\hline 5 & $=$ & $20,5^{\circ}$ & 2,1 & 61 & 57 & - & - & $\begin{array}{l}\text { Nach } 1 \text { Stunde kein Still- } \\
\text { stand. }\end{array}$ \\
\hline 6 & $=$ & $20^{\circ}$ & 2,1 & 69 & 36 & - & - & $\mathrm{dg} l$ \\
\hline 7 & $=$ & $25^{0}$ & 2,8 & 88 & 21 & $\tilde{5} 1$ & $\therefore$ & $\begin{array}{l}\text { Nach } 1 \text { Stunde kein compl. } \\
\text { Stillstand. }\end{array}$ \\
\hline 8 & $=$ & $20^{\circ}$ & 2,8 & 60 & 25 & 53 & - & dgl. \\
\hline 9 & $=$ & $20^{0}$ & 2,8 & 63 & 27 & 44 & 一 & dgl. \\
\hline 10 & $=$ & $20^{\circ}$ & 2,8 & 55 & 45 & - & - & $\begin{array}{l}\text { Nach } 1 \text { Stunde kein Still- } \\
\text { stand. }\end{array}$ \\
\hline 11 & $=$ & $22,5^{0}$ & $3, \overline{5}$ & 73 & 14 & 26 & - & $\begin{array}{l}\text { Nach } 1 \text { Stunde kein compl. } \\
\text { Stillstand. }\end{array}$ \\
\hline 12 & $=$ & $24^{\circ}$ & 3,5 & 75 & 15 & 24 & 49 & \\
\hline 13 & $=$ & $20,5^{\circ}$ & 3,5 & 52 & 18 & 37 & - & $\begin{array}{l}\text { Nach 1. Stunde kein compl. } \\
\text { Stillstand. }\end{array}$ \\
\hline
\end{tabular}

1) Es warden bis $12 \mathrm{mg}$ Fröschen subcutan beigebracht; ohne dass irgend eine Wirkung ersichtlich war. 


\begin{tabular}{|c|c|c|c|c|c|c|c|c|}
\hline $\begin{array}{l}\stackrel{\bar{\Phi}}{\Xi} \\
\stackrel{\Xi}{\Xi} \\
Z\end{array}$ & 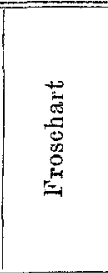 & 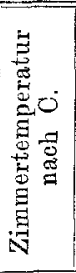 & 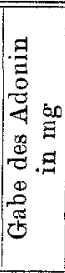 & 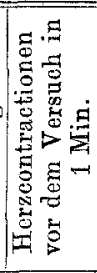 & 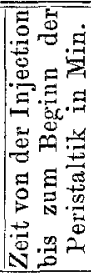 & 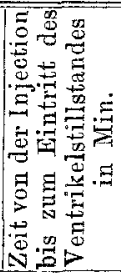 & 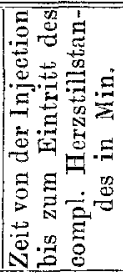 & Bemerkungen \\
\hline 14 & R. eseul & $20,5^{0}$ & 3,5 & 66 & - & - & - & $\begin{array}{l}\text { Nach } 1 \text { Stunde kein Still- } \\
\text { stand. Peristaltik undeutlich. }\end{array}$ \\
\hline 15 & $=$ & $25^{0}$ & 4,2 & 74 & 9 & 15 & 43 & \\
\hline 16 & $=$ & $22,5^{0}$ & 4,2 & 76 & 11 & 31 & 53 & \\
\hline 17 & $=$ & $23^{0}$ & 4,2 & 77 & 14 & 35 & - & $\begin{array}{l}\text { Nach } 1 \text { Stonde kein compl. } \\
\text { Stillstand. }\end{array}$ \\
\hline 18 & $=$ & $22^{\circ}$ & 4,2 & 74 & - & 28 & 60 & \\
\hline 19 & $=$ & $22^{\circ}$ & 4,2 & 77 & 10 & 31 & - & $\begin{array}{l}\text { Nach } 1 \text { Stunde kein compl. } \\
\text { Stillstand. }\end{array}$ \\
\hline 20 & $=$ & $22^{\circ}$ & 5,0 & 68 & 7 & 18 & 36 & \\
\hline 21 & $=$ & $23^{\circ}$ & 5,0 & 72 & 9 & 30 & 45 & \\
\hline 22 & $=$ & $24^{0}$ & 5,0 & 89 & 5 & 18 & 34 & \\
\hline 23 & $=$ & $22^{\circ}$ & 5,6 & 70 & 5 & 35 & 49 & \\
\hline 24 & $=$ & $23^{\circ}$ & 5,6 & 75 & 6 & 19 & 39 & \\
\hline 25 & $=$ & $23^{\circ}$ & 7,0 & 69 & 4 & 18 & 32 & \\
\hline 26 & R. temp. & $23^{\circ}$ & 0,5 & 77 & 17 & - & - & $\begin{array}{l}\text { Nach } 1 \text { Stunde kein Still- } \\
\text { stand. }\end{array}$ \\
\hline $2 \bar{\imath}$ & $=$ & $22^{\circ}$ & 1,0 & 82 & 13 & - & - & $\mathrm{dgl}$ \\
\hline 28 & $=$ & $22^{0}$ & 2,0 & 76 & 7 & 19 & - & $\begin{array}{l}\text { Nach 1 Stunde kein compl. } \\
\text { stillstand. }\end{array}$ \\
\hline 29 & $=$ & $24^{0}$ & 3,0 & 67 & 7 & 11 & -- & dgl \\
\hline 30) & $=$ & $23^{0}$ & 3,5 & 60 & 8 & 10 & - & dgl. \\
\hline 31 & $=$ & $23^{0}$ & 3,5 & 65 & 5 & 6 & 30 & \\
\hline 32 & $=$ & $24^{0}$ & 4,0 & 82 & 4 & 8 & 21 & \\
\hline 33 & $=$ & $24^{0}$ & 5,0 & 89 & 4 & 6 & 10 & \\
\hline
\end{tabular}

Man sieht aus dieser Zusammenstellung, dass die minimale Gabe des Adonin zur Herbeiführung des systolischen Herzstillstandes bei Temporaria $31 / 2-4 \mathrm{mg}$, bei Esculenta etwas mehr $(4-5 \mathrm{mg})$ beträgt. Controlversuche mit dem Merck'sehen Adonidin ergaben, dass schon weniger als $0,2 \mathrm{mg}$ genügte, um denselben Effect zu erzielen. Nach den Untersuchungen Cervello's wird dazu $0,15 \mathrm{mg}$ erfordert. Demzufolge wirkt das Adonin über $20 \mathrm{mal}$ sehwächer, als das Ad onidin.

Die muskellähmende Wirkung, die den Stoffen der Digitalingruppe eigenthümlich ist, tritt auch beim Adonin, allerdings erst nach grösseren Dosen, unverkennbar zu Tage.

In Bezug auf das Verhalten des Blutdrucks kann man Folgendes angeben:

Am Kaninchen wird der Blutdruck nach $30-50 \mathrm{mg}$ pro Kilo Körpergewicht bei intravenöser Injection auffallend erhöht. Bei geringeren Gaben findet man zwar den Herzschlag verstärkt und leicht 
Ueber den wirksamen Bestandtheil der Adonis amurensis Reg. et Radd. 305

verlangsamt, der Blutdruck aber steigt nur unbedeutend oder gar nicht. Wir lassen als Belege folgende Protokolle folgen:

1. Kaninchen von $1990 \mathrm{~g}$. Linke Carotis in Verbindung mit dem Manometer des Kymographions. Gabe des eingespritzten Adonin $=60,5 \mathrm{mg}$.

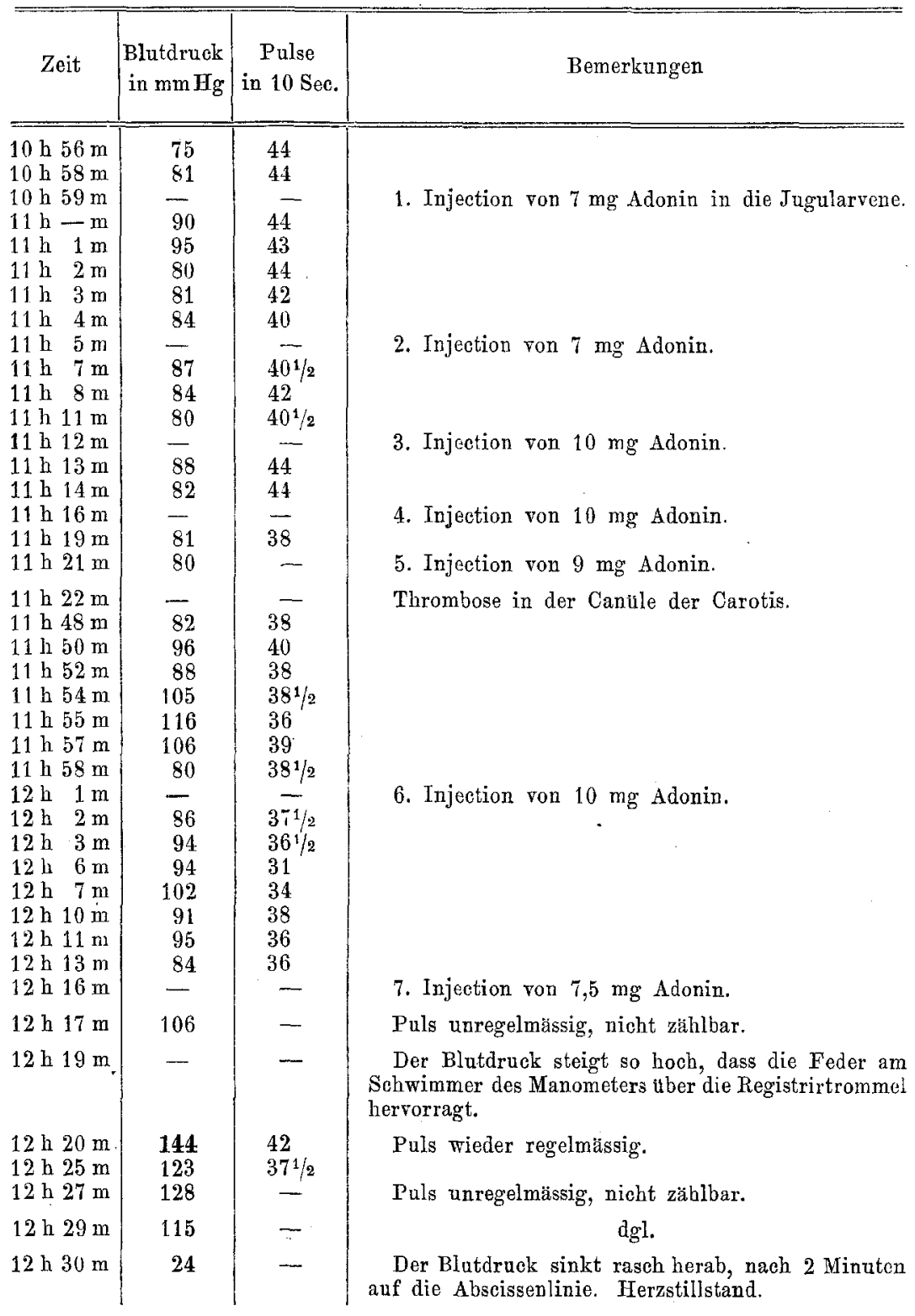


2. Kaninchen von $1650 \mathrm{~g}$. Linke Carotis mit dem Manometer verbunden. Narkose durch Urethan $(2 \mathrm{~g})$. Gabe des Adonin $=84 \mathrm{mg}$.

\begin{tabular}{|c|c|c|c|}
\hline Datum & $\begin{array}{l}\text { Blutdruek } \\
\text { in } \mathrm{mm} \mathrm{Hg}\end{array}$ & $\begin{array}{l}\text { Pulse } \\
\text { in } 10 \text { Sec. }\end{array}$ & Bemerkungen \\
\hline $3 \mathrm{~h} 5 \mathrm{~m}$ & 90 & 56 & \\
\hline $3 \mathrm{~h} 8 \mathrm{~m}$ & 90 & 56 & \\
\hline $3 \mathrm{~h} 10 \mathrm{~m}$ & 93 & 56 & 1. Injection von $15 \mathrm{mg}$ Adonin in die Jugularvene. \\
\hline $3 \mathrm{~h} 11 \mathrm{~m}$ & 95 & 53 & \\
\hline $3 \mathrm{~h} 12 \mathrm{~m}$ & 98 & 51 & \\
\hline $\begin{array}{l}3 \mathrm{~h} 14 \mathrm{~m} \\
3 \mathrm{~h} 15 \mathrm{~m}\end{array}$ & 96 & $\begin{array}{l}30 \\
49\end{array}$ & \\
\hline $3 \mathrm{~h} 16 \mathrm{~m}$ & 95 & 50 & 2. Injection von $15 \mathrm{mg}$ Adonin. \\
\hline $3 \mathrm{~h} 17 \mathrm{~m}$ & 95 & 48 & \\
\hline $3 \mathrm{~h} 18 \mathrm{~m}$ & 96 & 48 & \\
\hline $3 \mathrm{~h} 20 \mathrm{~m}$ & $\begin{array}{l}94 \\
91\end{array}$ & $\begin{array}{l}471 / 2 \\
471_{2}^{\prime}\end{array}$ & \\
\hline & $\begin{array}{l}91 \\
88\end{array}$ & $\begin{array}{l}47^{\prime} \cdot 2 \\
48\end{array}$ & \\
\hline $\begin{array}{l}3 \mathrm{~h} 25 \mathrm{~m} \\
3 \mathrm{~h} 26 \mathrm{~m}\end{array}$ & 90 & 49 & \\
\hline $3 \mathrm{~h} 27 \mathrm{~m}$ & 94 & 48 & 3. Injection von $12 \mathrm{mg}$ Adonin. \\
\hline $3 \mathrm{~h} 29 \mathrm{~m}$ & 98 & 49 & \\
\hline $3 \mathrm{~h} 30 \mathrm{~m}$ & 100 & 49 & \\
\hline $3 \mathrm{~h} 31 \mathrm{~m}$ & 102 & 52 & \\
\hline $3 \mathrm{~h} 32 \mathrm{~m}$ & 103 & $521 / 2$ & \\
\hline $3 \mathrm{~h} 35 \mathrm{~m}$ & 99 & $531 / 2$ & \\
\hline $3 \mathrm{~h} 36 \mathrm{~m}$ & 100 & 48 & 4. Injection von $8 \mathrm{mg}$ Adonin. \\
\hline $3 \mathrm{~h} 37 \mathrm{~m}$ & 104 & $51^{1}, 2$ & \\
\hline $3 \mathrm{~h} 38 \mathrm{~m}$ & $\begin{array}{r}104 \\
98\end{array}$ & $52^{1} 2$ & \\
\hline $\begin{array}{l}3 \mathrm{~h} 40 \mathrm{~m} \\
3 \mathrm{~h} 42 \mathrm{~m}\end{array}$ & $\begin{array}{l}98 \\
90\end{array}$ & $\begin{array}{l}52 \\
49\end{array}$ & \\
\hline $3 \mathrm{~h} 43 \mathrm{~m}$ & 88 & 50 & \\
\hline $3 \mathrm{~h} 45 \mathrm{~m}$ & 90 & 54 & \\
\hline $3 \mathrm{~h} 47 \mathrm{~m}$ & 86 & 54 & 5. Injection von $6 \mathrm{mg}$ Adonin. \\
\hline $3 \mathrm{~h} 50 \mathrm{~m}$ & 84 & $55^{1 / 2}$ & \\
\hline $3 \mathrm{~h} 54 \mathrm{~m}$ & $\begin{array}{l}83 \\
80\end{array}$ & - & Puls klein, nicht zählbar. \\
\hline $\begin{array}{l}3 \mathrm{~h} 56 \mathrm{~m} \\
3 \mathrm{~h} 58 \mathrm{~m}\end{array}$ & $\begin{array}{l}80 \\
79\end{array}$ & - & $\begin{array}{l}\text { dgl. } \\
\text { dgl. }\end{array}$ \\
\hline $4 \mathrm{~h} 1 \mathrm{~m}$ & 78 & - & dgl. \\
\hline $4 \mathrm{~h} \quad 4 \mathrm{~m}$ & 76 & - & dgl. \\
\hline $4 \mathrm{~h} 9 \mathrm{~m}$ & 78 & - & dgl. \\
\hline $4 \mathrm{~h} 14 \mathrm{~m}$ & 74 & - & dgl. \\
\hline $4 \mathrm{~h} 17 \mathrm{~m}$ & 80 & 40 & $\begin{array}{l}\text { 6. Injection von } 20 \mathrm{mg} \text { Adonin. Puls kräftig, aber } \\
\text { nicht regelmässig. }\end{array}$ \\
\hline $\begin{array}{l}4 \mathrm{~h} 19 \mathrm{~m} \\
4 \mathrm{~h} 20 \mathrm{~m}\end{array}$ & $\begin{array}{l}54 \\
63\end{array}$ & 41 & $\begin{array}{l}\text { Puls unregelmässig. } \\
\text { Puls nicht zählbar. }\end{array}$ \\
\hline $\begin{array}{l}4 \mathrm{~h} 20 \mathrm{~m} \\
4 \mathrm{~h} 21 \mathrm{~m}\end{array}$ & 68 & $40^{1 / 2}$ & $\begin{array}{l}\text { Puis nicht zählbar. } \\
\text { Puls unregelmässig. }\end{array}$ \\
\hline $4 \mathrm{~h} 22 \mathrm{~m}$ & 71 & 45 & $\mathrm{dgl}$. \\
\hline $4 \mathrm{~h} 24 \mathrm{~m}$ & 76 & $46^{1 / 2}$ & Puls wieder regelmässig. \\
\hline $4 \mathrm{~h} 25 \mathrm{~m}$ & 80 & $40^{\circ}$ & \\
\hline $4 \mathrm{~h} 26 \mathrm{~m}$ & 82 & 48 & \\
\hline $4 \mathrm{~h} 27 \mathrm{~m}$ & 78 & 44 & 7. Injection von $4 \mathrm{mg}$ Adonin. \\
\hline $4 \mathrm{~h} 29 \mathrm{~m}$ & 77 & 44 & \\
\hline $4 \mathrm{~h} 31 \mathrm{~m}$ & 82 & 49 & \\
\hline $4 \mathrm{~h} 32 \mathrm{~m}$ & 88 & 50 & 8. Injection von $4 \mathrm{mg}$ Adonin. \\
\hline $4 \mathrm{~h} 33 \mathrm{~m}$ & 93 & 47 & \\
\hline $4 \mathrm{~h} 34 \mathrm{~m}$ & 101 & 49 & \\
\hline $4 \mathrm{~h} 35 \mathrm{~m}$ & 109 & $47^{1 / 2}$ & \\
\hline
\end{tabular}


Ueber den wirksamen Bestandtheil der Adonis amurensis Reg. et Radd. 307

\begin{tabular}{|c|c|c|c|}
\hline Zeit & $\left|\begin{array}{l}\text { Blutdruck } \\
\text { in } \mathrm{mm} \mathrm{Hg}\end{array}\right|$ & $\begin{array}{c}\text { Pulse } \\
\text { in } 10 \text { See. }\end{array}$ & Bemerkungen \\
\hline $\begin{array}{l}4 \mathrm{~h} 36 \mathrm{~m} \\
4 \mathrm{~h} 37 \mathrm{~m} \\
4 \mathrm{~h} 38 \mathrm{~m} \\
4 \mathrm{~h} 39 \mathrm{~m} \\
4 \mathrm{~h} 40 \mathrm{~m} \\
4 \mathrm{~h} 42 \mathrm{~m} \\
4 \mathrm{~h} 49 \mathrm{~m}\end{array}$ & $\begin{array}{r}124 \\
141 \\
\mathbf{1 5 0} \\
144 \\
130 \\
114 \\
89\end{array}$ & $\begin{array}{l}43 \\
42 \\
42^{1 / 2} \\
43^{1 / 2} \\
42 \\
45 \\
-\end{array}$ & $\begin{array}{l}\text { Puls aussetzend. } \\
\text { Puls klein, nicht zählbar. Der Blutdruck fällt } \\
\text { dann schnell auf die Abscisse. }\end{array}$ \\
\hline
\end{tabular}

Vergleichende Untersuchungen mit dem Merck'schen Adonidin führten zu dem Resultat, dass Dosen unter $2 \mathrm{mg}$ pro Kilo Körpergewicht bereits im Stande waren, eine mächtige Blutdruckerhöhung zu bewirken. ${ }^{1}$ )

1. Kaninchen von $2400 \mathrm{~g}$. Linke Carotis mit dem Quecksilbermanometer in Verbindung. Gabe des Adonidin Merck's $=4 \mathrm{mg}$.

\begin{tabular}{|c|c|c|c|}
\hline Zeit & $\begin{array}{l}\text { Blutdruck } \\
\text { in } \mathrm{mm} \mathrm{Hg}\end{array}$ & $\begin{array}{c}\text { Pulse } \\
\text { in } 10 \text { See. }\end{array}$ & Bemerkungen \\
\hline $\begin{array}{l}1 \mathrm{~h} 19 \mathrm{~m} \\
1 \mathrm{~h} 20 \mathrm{~m}\end{array}$ & 94 & $\begin{array}{l}46 \\
471 / .\end{array}$ & \\
\hline $1 \mathrm{~b} 21 \mathrm{~m}$ & 102 & $42^{1 / 2}$ & 1. Injection ron $0,4 \mathrm{mg}$ Adonidin in die Jugularvene. \\
\hline $1 \mathrm{~h} 22 \mathrm{~m}$ & 102 & $40^{1 / 2}$ & 2. Injection von $0,3 \mathrm{mg}$ Adonidin. \\
\hline $1 \mathrm{~h} 23 \mathrm{~m}$ & 104 & 41 & \\
\hline $1 \mathrm{~h} 24 \mathrm{~m}$ & 103 & $361 / 2$ & 3. Injection von $0,4 \mathrm{mg}$ Adonidin. \\
\hline $1 \mathrm{~h} 25 \mathrm{~m}$ & 107 & 37 & \\
\hline $1 \mathrm{~h} 26 \mathrm{~m}$ & 110 & 34 & 4. Injection von $0,4 \mathrm{mg}$ Adonidin. \\
\hline $1 \mathrm{~h} 25 \mathrm{~m}$ & 107 & $371 / 2$ & \\
\hline $1 \mathrm{~h} 29 \mathrm{~m}$ & 160 & - & 5. Injection von $0,5 \mathrm{mg}$ Adonidin. \\
\hline $1 \mathrm{~b} 30 \mathrm{~m}$ & 170 & 28 & Puls unregelmässig. \\
\hline $1 \mathrm{~h} 31 \mathrm{~m}$ & 143 & - & Puls klein, nicht zählbar. \\
\hline $1 \mathrm{~h} 32 \mathrm{~m}$ & 132 & - & dge. \\
\hline $1 \mathrm{~h} 33 \mathrm{~m}$ & 110 & ... & dgl. \\
\hline $1 \mathrm{~h} 34 \mathrm{~m}$ & 90 & - & dgl. \\
\hline $1 \mathrm{~h} 36 \mathrm{~m}$ & 100 & - & $\begin{array}{l}\text { 6. Injection vou } 0,4 \mathrm{mg} \text { Adonidin. Puls klein, nicht } \\
\text { 2ähibar. }\end{array}$ \\
\hline $1 \mathrm{~h} 38 \mathrm{~m}$ & 93 & - & Puls klein, nicht zählbar. \\
\hline $1 \mathrm{~h} 40 \mathrm{~m}$ & 97 & - & dgl. \\
\hline $1 \mathrm{~h} 50 \mathrm{~m}$ & 85 & 43 & Puls wieder regelmässig. \\
\hline $2 \mathrm{~b}-\mathrm{m}$ & 90 & 40 & \\
\hline $2 \mathrm{~h} 4 \mathrm{~m}$ & - & - & 7. Injection von $0,8 \mathrm{mg}$ Adonidin. \\
\hline $2 \mathrm{~h} 5 \mathrm{~m}$ & 106 & 43 & \\
\hline $2 \mathrm{~h} 6 \mathrm{~m}$ & 113 & 45 & \\
\hline $2 \mathrm{~h} 7 \mathrm{~m}$ & 120 & $42^{1 / 2}$ & \\
\hline $2 \mathrm{~L} 9 \mathrm{~m}$ & 127 & 38 & \\
\hline $2 \mathrm{~b} 10 \mathrm{~m}$ & 120 & 38 & \\
\hline $2 \mathrm{~h} 11 \mathrm{~m}$ & 147 & - & 8. Inject. von $0,8 \mathrm{mg}$ Adonidin. Puls unregelmässig. \\
\hline $2 \mathrm{~h} 12 \mathrm{~m}$ & 102 & - & Pulsschläge klein, nieht zählbar. \\
\hline $2 \mathrm{~h} 13 \mathrm{~m}$ & 47 & - & dgl. \\
\hline $2 \mathrm{~h} 14 \mathrm{~m}$ & - & -- & Der Blutdruck fallt auf die Nullinie. Herzstillstand. \\
\hline
\end{tabular}

1) Vgl. auch Cervello, l. c. S. 243. 
2. Kaninchen von $2150 \mathrm{~g}$. Rechte Carotis mit dem Manometer in Verbindung. Narkose durch Urethan $(2 \mathrm{~g})$. Gabe des Adonidin Merck $=3,9 \mathrm{mg}$.

\begin{tabular}{|c|c|c|c|}
\hline Zeit & $\begin{array}{l}\text { Blutdruek } \\
\text { in } \mathrm{mm} \mathrm{Hg}\end{array}$ & $\begin{array}{c}\text { Pulse } \\
\text { in } 10 \mathrm{Sec} .\end{array}$ & Bemerkungen \\
\hline $\begin{array}{l}11 \mathrm{~h} 55 \mathrm{~m} \\
11 \mathrm{~h} 56 \mathrm{~m}\end{array}$ & $\begin{array}{l}73 \\
72\end{array}$ & $\begin{array}{l}48^{1} / 2 \\
48\end{array}$ & \\
\hline $11 \mathrm{~h} 57 \mathrm{~m}$ & 78 & $471 / 2$ & 1. Injeotion von $0,65 \mathrm{mg}$ Adonin in die Jugularvene. \\
\hline $11 \mathrm{~h} 59 \mathrm{~m}$ & 80 & 48 & \\
\hline $12 \mathrm{~h}-\mathrm{m}$ & 74 & 48 & \\
\hline $12 \mathrm{~h} 1 \mathrm{~m}$ & 79 & 48 & 2. Injection von $0,65 \mathrm{mg}$ Adonidin. \\
\hline $12 \mathrm{~h} \quad 3 \mathrm{~m}$ & 73 & 48 & \\
\hline $12 \mathrm{~h} 4 \mathrm{~m}$ & 72 & $431 / 2$ & 3. Injection von $0,65 \mathrm{mg}$ Adonidin. \\
\hline $12 \mathrm{~h} 5 \mathrm{~m}$ & 76 & 46 & \\
\hline $12 \mathrm{~h} 6 \mathrm{~m}$ & 62 & 47 & \\
\hline $12 \mathrm{~h} 7 \mathrm{~m}$ & 42 & 46 & \\
\hline $12 \mathrm{~h} 8 \mathrm{~m}$ & 40 & - & 4. Inject. von $0,65 \mathrm{mg}$ Adonidin. Puls nicht zähibar. \\
\hline $12 \mathrm{~h} 10 \mathrm{~m}$ & 70 & - & Puls nicht zälılbar. \\
\hline $12 \mathrm{~h} 11 \mathrm{~m}$ & 86 & - & dgl. \\
\hline $12 \mathrm{~h} 12 \mathrm{~m}$ & 92 & & \\
\hline $12 \mathrm{~h} 13 \mathrm{~m}$ & 84 & 40 & \\
\hline $12 \mathrm{~h} 14 \mathrm{~m}$ & 68 & $43^{1 / 2}$ & \\
\hline $12 \mathrm{~h} 16 \mathrm{~m}$ & 35 & 44 & \\
\hline $12 \mathrm{~h} 17 \mathrm{~m}$ & 33 & $47^{1 / 2}$ & 5. Injection von $0,65 \mathrm{mg}$ Adonidin. \\
\hline $12 \mathrm{~h} 18 \mathrm{~m}$ & 47 & 48 & \\
\hline $12 \mathrm{~h} 20 \mathrm{~m}$ & 52 & 45 & \\
\hline $12 \mathrm{~h} 21 \mathrm{~m}$ & 63 & 44 & \\
\hline $12 \mathrm{~h} 23 \mathrm{~m}$ & 86 & - & Puls nioht zählbar. \\
\hline $12 \mathrm{~h} 25 \mathrm{~m}$ & 96 & 38 & \\
\hline $12 \mathrm{~h} 26 \mathrm{~m}$ & 110 & $40^{1 / 2}$ & \\
\hline $12 \mathrm{~h} 27 \mathrm{~m}$ & 126 & $43^{\prime}$ & \\
\hline $12 \mathrm{~h} 28 \mathrm{~m}$ & 144 & 42 & \\
\hline $12 \mathrm{~h} 29 \mathrm{~m}$ & 140 & $31^{1 / 2}$ & . \\
\hline $12 \mathrm{~h} 30 \mathrm{~m}$ & 123 & 42 & \\
\hline $12 \mathrm{~h} 31 \mathrm{~m}$ & 115 & 45 & \\
\hline $12 \mathrm{~h} 32 \mathrm{~m}$ & 96 & 39 & \\
\hline $1.2 \mathrm{~h} 33 \mathrm{~m}$ & 71 & - & 6. Injection von $0,65 \mathrm{mg}$ Adonidin. \\
\hline $12 \mathrm{~h} 34 \mathrm{~m}$ & 46 & - & Puls nicht zählbar. \\
\hline $12 \mathrm{~h} 35 \mathrm{~m}$ & 58 & - & dgl. \\
\hline $12 \mathrm{~h} 36 \mathrm{~m}$ & 80 & - & dgl. \\
\hline $12 \mathrm{~h} 37 \mathrm{~m}$ & 90 & - & $\mathrm{dgl}$ \\
\hline $12 \mathrm{~h} 38 \mathrm{~m}$ & 99 & - & dgl. \\
\hline $12 \mathrm{~h} 39 \mathrm{~m}$ & 104 & - & dgl. \\
\hline $12 \mathrm{~h} 41 \mathrm{~m}$ & 112 & - & dgl. \\
\hline 12 h. $43 \mathrm{~m}$ & 120 & - & dgl. \\
\hline $12 \mathrm{~h} 45 \mathrm{~m}$ & 114 & - & dgl. \\
\hline $12 \mathrm{~h} 48 \mathrm{~m}$ & 92 & - & dgl. \\
\hline $12 \mathrm{~h} 50 \mathrm{~m}$ & 60 & 46 & \\
\hline $12 \mathrm{~h} 53 \mathrm{~m}$ & 52 & 46 & $\begin{array}{l}\text { Der Blutdruek fallt raseh berab und naoh einigen } \\
\text { Minuten tritt Herzstillstand ein. }\end{array}$ \\
\hline
\end{tabular}

Es erhellt aus diesen Versuchen, dass das Adonin aucb auf das Säugethier viel sehwächer wirkt, wie das Adonidin. Ebenso scheint der gesunde Mensch ziemlich hobe Dosen von Adonin $\mathrm{zu}$ ertragen. Ich habe an mir selbst einige Versuche an- 
gestellt: einmal babe ich $30 \mathrm{mg}$ per os in refracta dosi binnen 24 Stunden genommen, ein anderes Mal dieselbe Menge pro dosi. Beide Male vermisste ich irgend welche nennenswerthe Veränderung sowohl des Pulses, als auch des Allgemeinbefindens. Ich konnte jedoch leider nicht zu noch böberen Dosen greifen, daran war ich aus Mangel an Material verhindert.

Das Extract der Adonis amurensis wirkt ganz analog wie das reine Adonin. In dieser Hinsicht habe ich an mir zwei Experimente gemacht.

1. Ein heisser Aufguss von $4 \mathrm{~g}$ Wurzel in $100 \mathrm{~g}$ Wasser wurde innerbalb 6 Stunden getrunken. Der Puls ging darnach nur um einige Schläge herab, obne sonst in eigenthümlicher Weise verändert zu werden.

2. Dagegen brachte ein Infus von $6,0: 100,0$, binnen 4 Stunden genommen, charalsteristische Symptome hervor. Es war 4 Uhr Abends, als ich den letzten Theil der Lösung austrank. Da war mein Puls, der sonst 71 in 1 Minute zählte, bereits auf 64 gesunken. Er ging inmer mehr herab, zugleich voller werdend, und Nachts ura $12 \mathrm{Ubr}$ betrug seine Frequenz 52 Schläge. Das Allgemeinbefinden war nicht gestört. Am anderen Morgen fühite ich Uebelkeit und erbrach 2 mal. Der Appetit schwand und allgemeine Muidigkeit trat ein. Der Puls wurde etwas frequenter $(70-80)$, zugleich schwächer und zählte am Abend, zu welcher Zeit ich noch einige Male erbrach, 80-90 Schläge, war ziemlich klein and unregelmässig. Am 3. Tag Puls 60-65, unregelmässig, intermittirend je 2-3 Schläge, aber sonst in voller Spannung. Ich bekam wieder Appetit. Erst am 4. Tag wurde der Puls normal.

Einige therapeutische Versuche, die ich bis jetzt gemacht habe, scheinen fur die praktische Anwendbarkeit der Pflanze zu sprechen. Ich exlaube mir als Beleg eine Krankengeschichte in aller Kürze mitzutheilen.

K. Nakakita, ein 27 jäbr. Mann. Kakke (Beri-Beri).

An amnese. Seit Ende 1889 Symptome der Kakke, Sensibilitätsund Motilitätsstörung und Oedem.

Status praesens (15. September 1890). Kräftiger Körperban. Allgemeines Anasarka. Lungen normal. Herz dilatirt: die Dämpfung beginnt am unteren Rand der 2. Rippe, reicht rechts bis zur Mitte des sternum, links $2 \mathrm{~cm}$ über die Mamillarlinie, Spitzenstoss im 6. Intercostalraum. Herztöne rein. Am Abdomen nichts Besonderes. Patellarsehnenreflex total verschwunden. Puls 108, regelmässig, aber ziemlich klein. Urin normal, Tagesmenge $1200 \mathrm{ccm}$.

Verordnung: Inf. rad. Adon. amur. $(6,0) 100$ (Tagesdose). 
310 XXIll. INoko, Ueber den wirksamen Bestandtheil der Adonis u. s. w.

Verlauf. 16. September Morgens einmal Erbrechen. Allgemeine Muidigkeit. Puls 52, aussetzend alle 5-6 Schläge, Urinmenge vermindert, $850 \mathrm{ccm}$ pro die. - 17. September. Puls 40, voll, aber unregelmässig. Appetit schlecht, einmal Erbrechen. - 18. September. Puls 42, voll. Urinmenge vermehrt, $1380 \mathrm{ccm}$. - 19. September. Urinmenge $1410 \mathrm{ccm}$. - 20. September. Appetit wieder besser. Oedem mindert sich zusehends. Puls 40, regelmässig, voll. Urinmenge $1380 \mathrm{ccm}$. - 21. September. Urinmenge $1610 \mathrm{ccm}$ - - 22. September. Puls 64, ganz kräftig. Allgemeinbefinden bessert sich. Urinmenge $1820 \mathrm{ccm}-23$. September. Urinmenge $1710 \mathrm{ccm}$. - 24. September. Puls 50. Oedem der Beine bedentend abgenommen. Urinmenge $1820 \mathrm{ccm}$. - 25. September. Urinmenge $1650 \mathrm{ccm}$. - 26. September. Urinmenge $2010 \mathrm{ccm}$. - 27. September. Puls 50, kräftig. Nur noch wenig Oedem zu sehen. - Im weiteren Verlauf nahm der Puls allmählich wieder zu.

Fassen wir die Resultate zusammen, so ist der wirksame Bestandtheil der Adonis amurensis nicht das Adonidin, sondern das Adonin und dürfte wohl dieser Körper oder die Pflanze therapeutisch verwerthet werden.

Tokio, im December 1890. 